\title{
Characterization of JAK2 V617F (1849 G > T) Mutation in Cervical Cancer Related to Human Papillomavirus and Sexually Transmitted Infections
}

\author{
Masoumeh Abdolmaleki ${ }^{1}$, Amir Sohrabi ${ }^{2}$ \\ 'Department of Biology, East Tehran Branch, Islamic Azad University, ${ }^{2}$ Department of Molecular Biology, Research Center of Health Reference \\ Laboratory, Ministry of Health and Medical Education, Tehran, Iran
}

\begin{abstract}
Background: Human papillomavirus (HPV) genotypes that infect the genital tract play a main etiologic role in cervical cancer progression. Other environmental factors, such as sexually transmitted diseases and the host genetic pattern, contribute to infection persistence of the uterus and cervical epithelium in sustaining their malignancy. The Janus kinase 2 is a non-receptor tyrosine kinase in cell signaling process of tumor genesis. In the present study, JAK2 V167F mutation was distinguished in women with sexually transmitted infections, such as Herpes simplex virus 2, Chlamydia trachomatis and Mycoplasma genitalium and cervical cancer.

Methods: This case-control survey was performed on 195 liquid based cytology of women specimens. Fifty, 98 , and 47 samples were from women with known cervical cancer, HPV positive and HPV negative, respectively. Single nucleotide polymorphism analysis, sexually transmitted infections detection and HPV genotyping were carried out using approved PCR- RFLP, in-house multiplex TaqMan Real Time PCR and the reverse dot blot hybridization assay.

Results: HPVs 6, 16, 18, 11, 31, and 51 were the most common genotypes. The prevalence rate of multiple HPV genotypes was $46.0 \%$ to $10.1 \%$. Analysis of JAK2 V617F (1849 G > T) showed that prevalence of mutation was GG (65.1\%), GA (34.9\%), and TT (0\%), respectively. There were no statistically significant differences between this mutation and variables of population survey $(P \geq 0.05)$. Conclusions: The molecular epidemiology study on the genetic polymorphisms, i.e., JAK2 V617F and other single nucleotide polymorphisms as a diagnostic tool is necessary for cancer screening and prophylactic programs.
\end{abstract}

(J Cancer Prev 2018;23:82-86)

Key Words: Human papillomavirus, Sexually transmitted infections, Janus kinase 2, Polymorphism, Cervical cancer, Iran

\section{INTRODUCTION}

Cervical cancer is ranked second across the top five women malignancies in worldwide. Multiple factors, such as human papillomavirus (HPV) and sexually transmitted infections (STIs) followed by the activity of oncogenes and environmental factors, are linked to complex diseases like cervical precancerous and cancerous. ${ }^{1,2}$ Persistent high risk HPV genotypes with other genital pathogens are essential for enhancing the susceptibility to genital malignancies. ${ }^{3-7}$

Some potential cancer antigens and biomarkers, such as
CA-125, CA-19-9, epigenetic and genetic alterations and polymorphisms in tumor suppressor genes are utilized in prognostic and diagnostic exposures in clinical management of women. ${ }^{8-11}$

It seems that $50 \%$ to $90 \%$ of early cervical neoplasia items regress spontaneously, although host genetic factors especially the immune system plays an important role in preventing genital disorders. ${ }^{12-14}$ The single nucleotide polymorphisms in immune modulating genes like JAK-STAT pathway are associated with a variety of cancers. STAT activation needs tyrosine phosphorylation by a receptor associated Janus kinase (JAK). JAKs particularly JAK2 are non-receptor tyrosine kinase that plays a

Received March 21, 2018, Revised May 27, 2018, Accepted June 7, 2018

Correspondence to: Amir Sohrabi

Department of Molecular Biology, Research Center of Health Reference Laboratory, Ministry of Health and Medical Education, 48 Keykhosro Shahrokh Alley, Zartoshtian Alley, Hafez Avenue, P.O. Box: 1131636111, Tehran, Iran

Tel: +98-2166728112 (Ext.114), Fax: +98-2166728121, E-mail: sohrabi58@gmail.com, ORCID: Amir Sohrabi, https://orcid.org/0000-0001-5867-3636

Copyright (C) 2018 Korean Society of Cancer Prevention

(c) This is an Open Access article distributed under the terms of the Creative Commons Attribution Non-Commercial License (http://creativecommons.org/icenses/by-nc/4.0) which permits unrestricted non-commercial use, distribution, and reproduction in any medium, provided the original work is properly cited. 
significant role in cell signaling process of tumor genesis. Genital co-infections are simultaneously proposed to increase susceptibility to cervical cancer. ${ }^{1418}$

In the present molecular epidemiology survey, the most common sexually transmitted pathogens, e.g., Chlamydia trachomatis, HSV-2 and Mycoplasma genitalium and HPV as major agent of cervical malignancies were investigated in women with and at risk for cervical cancer. The JAK2 V617F (1849 G > T) mutation was selected based on the scientific databases which polymorphism is evaluated for probability of association with cervical cancer related to these STIs in Iranian subjects.

\section{MATERIALS AND METHODS}

\section{Study population}

A total of 195 liquid based cytology (LBCs) specimens were collected from women referred to Mohebe-Yas Hospital and private pathobiology laboratories, Tehran, Iran. Cervical scrapping and genital lesions with known cervical cancer (all the diagnoses were confirmed by histopathology examinations), HPV positive and healthy women (as negative control) were 50, 98, and 47, respectively. In order to meet ethical considerations, each cancer patient was informed about the objectives of study and signed a consent form before participating in the study. Other samples were archival specimens and necessary clinical data were recorded from medical documents.The LBCs were transported to the Reference Health Laboratory, Tehran, Iran in a proper storage area where they were stored at $-20^{\circ} \mathrm{C}$ until experimental phase.

\section{Statistical analysis}

In order to determine any significant relationship, an independent test (chi-square) was used between the variables $C$. trachomatis, HSV-2 and $M$. genitalium and HPV genotypes with cervical cancer and existence of JAK2 V617F (1849 G > T) polymorphism. A statistical test was performed at the significant level of 0.05 using IBM SPSS software ver. 23 (IBM Co., Armonk, NY, USA).

\section{Genome extraction}

Microbial and genomic DNA from LBCs specimens was extracted using the QIAamp DNA Mini kit (QIAGEN, Hilden, Germany) according to the manufacturer's instructions. ${ }^{19}$

\section{Human papillomavirus genotyping}

HPV detection and genotyping was performed by a reverse dot blot hybridization diagnostic kit using INNO-LiPA ${ }^{\circledR}$ HPV Genotyping Extra I\&II (Fujirebio, Tokyo, Japan), according to the previously described method. ${ }^{19}$

\section{Analysis of JAK2 V617F (1849 G > T) polymorphism}

Genome was analyzed by qualitative PCR and RFLP using gene specific primers. PCR amplification was carried out in a $25 \mu \mathrm{L}$ reaction volume containing $2 \times$ master mix red $1.5 \mathrm{mM} \mathrm{MgCl}_{2}$ (Ampliqon, Odense, Denmark), $10 \mathrm{pmol} / \mu \mathrm{L}$ of forward and reverse primer and $10 \mu \mathrm{L}$ of the extracted DNA as template. This reaction mixture was amplified at $94^{\circ} \mathrm{C}$ for 5 minutes and for 40 cycles in a thermal cycler (Techne, Staffordshire, UK) under the following conditions: $94^{\circ} \mathrm{C}$ for 45 seconds, $60^{\circ} \mathrm{C}$ for 45 seconds, $72^{\circ} \mathrm{C}$ for 50 seconds, plus a final extension step at $72^{\circ} \mathrm{C}$ for 10 minutes. Each experiment was performed with distilled water as a negative PCR control and patients with known genome as positive control. In RFLP analysis, $10 \mu \mathrm{L}$ of JAK2 PCR product was digested with $20 \mu \mathrm{L}$ restriction enzyme reaction containing $16 \mu \mathrm{L}$ sterile distilled water, $3 \mu \mathrm{L}$ enzyme buffer and Bsax $1(2,000 \mathrm{U} / \mu \mathrm{L})$ enzyme (New England Biolabs ${ }^{\circledR}$, Ipswich, MA, USA). The PCR-RFLP products were separated by electrophoresis on a $3 \%$ agarose gel, stained by Sybr safe (Invitrogen, Carlsbad, CA, USA), and were visualized under ultraviolet light along with a molecular weight marker (50 bp). In a JAK2 V617F (1849 G > T) PCR-RFLP reaction was expected to produce $364 \mathrm{bp}$ as undigest, 161 and 203 bp as wild type, 364, 203, $161 \mathrm{bp}$ as heterozygous and $364 \mathrm{bp}$ as homozygous. $^{20}$

\section{Qualitative TaqMan Real Time PCR Detection of sexually transmitted infections}

We developed primers and probes for $C$. trachomatis, HSV-2 and $M$. genitalium detection in TaqMan Real Time PCR reaction. The method data were described in previously document. ${ }^{19}$

\section{RESULTS}

In 148 out of 195 LBCs specimens, HPV s were detected for 28 HPV genotypes. HPVs 6 (35.13\%), 16 (32.43\%), 18 (21.62\%), 11 (9.46\%), 31 (9.46\%), and $51(9.46 \%)$ were the most common genotypes. The prevalence rate of multiple HPV genotypes was $46.0 \%$ to $10.1 \%$. The mean age of subjects was $35.5 \pm 10.13$ years with the age range of 19 to 70 years. The high HPV prevalence was observed in 25 to 35 years of age groups (32.8\%). The STIs outcomes detection using TaqMan Real Time PCR was 3 (1.54\%) for $M$. genitalium, 24 (12.3\%) for $C$. trachomatis and 1(0.5\%) for HSV-2. The details data of HPV genotyping and STIs aren't 
shown. ${ }^{19}$ The genotype distribution for JAK2 V617F (1849 G > T) polymorphism in women with cervical cancer, HPV positive and HPV negative groups are shown in Table 1. JAK2 genotype TT was not distinguished in all of subjects, although prevalence of the GG genotype was $127(65.1 \%)$ in case and control groups. The observed genotype frequencies among the STIs pathogens are summarized in Table 2. No significant difference was observed in genotype frequency between patients (cervical cancer, HPV positive and healthy women) for JAK2 polymorphism and STI pathogens $(P \geq 0.05)$.

\section{DISCUSSION}

HPV genotypes are certainly the major causes of genital malignancies particularly cervical cancer and precancerous lesions. There is growing evidence of HPV being a relevant factor in other anogenital cancers as well as head and neck cancers. ${ }^{21-23}$ The annual incidence of cervical cancer related to high risk-HPV genotypes in Iran is closed to 947 cases and mortality rate is 370 in 3.1 million women at risk for cervical cancer (female population aged $\geq 15$ years) ${ }^{24}$ Epigenetic and genetic polymorphisms patterns as diagnostic biomarker and HPV genotypes detection can be used for determining appropriate treatment strategy for early stages of cervical cancer and increase survival in cervical carcinogenesis. ${ }^{9.25}$ Persistent high risk-HPV genotypes with other STIs, environmental and host genetic factors may play critical roles in further malignant conversion of cervical epithelium and genital areas.

Analysis of JAK2 V617F (1849 G > T) polymorphism gene was assessed in women with and at risk for cervical cancer and genital disorders that only a few studies have been reported in the literature. Our results showed that the TT genotype was not detected in any study groups. The GG genotype was commonly observed in HPV negative women. Although, the highest rate of the GT genotype was recognized in women suffering from cervical cancer who were coinfected with HPVs and $C$. trachomatis. The SPSS ver. 23 statistical analysis showed that no significant difference was observed in $1849 \mathrm{G}>\mathrm{T}$ genotype frequency and STIs between the study population $(P \geq 0.05)$. The

Table 1. Distribution of JAK2 V617F poymorphism in women with STIs and cervical cancer

\begin{tabular}{|c|c|c|c|c|c|c|c|c|c|c|c|c|}
\hline \multicolumn{2}{|c|}{ Population study } & \multicolumn{3}{|c|}{$\begin{array}{c}\text { Women with cervical cancer } \\
\text { (50 cases) }\end{array}$} & \multicolumn{6}{|c|}{$\begin{array}{l}\text { Women without cervical cancer } \\
\text { (145 cases) }\end{array}$} & \multirow[t]{2}{*}{ Total } & \multirow[t]{2}{*}{$\begin{array}{c}\text { Test of } \\
\text { independence }\end{array}$} \\
\hline \multicolumn{2}{|c|}{ Patients categorizes } & \multicolumn{3}{|c|}{$\mathrm{CIN} \mathrm{I}=9$ cases; $\mathrm{CIN}$ II $=6$; $\mathrm{CIN}$ III $=35$} & \multicolumn{6}{|c|}{ HPV positive (98 cases); HPV negative (47 cases) } & & \\
\hline STIs pathogens & & $\mathrm{CT}$ & MG & HSV-2 & $\mathrm{CT}$ & MG & HSV-2 & $\mathrm{CT}$ & MG & HSV-2 & & \\
\hline STIs prevalence & & 7 Positive $^{a}$ & Undetected & Undetected & 7 & 2 & 1 & 10 & 1 & 0 & 28 & \\
\hline \multirow[t]{3}{*}{$\begin{array}{l}\text { JAK2 V617F } \\
\qquad(1849 \mathrm{G}>\mathrm{T})\end{array}$} & Wild type & 1 & 0 & 0 & 6 & 1 & 1 & 9 & 1 & 0 & 18 & $\begin{array}{l}\chi^{2}=0.232 \\
\text { (chi-square \& }\end{array}$ \\
\hline & Heterozygous & 6 & 0 & 0 & 1 & 1 & 0 & 1 & 0 & 0 & 9 & $\begin{array}{l}\text { Fisher's } \\
\text { exact test) }\end{array}$ \\
\hline & Homozygous & 0 & 0 & 0 & 0 & 0 & 0 & 0 & 0 & 0 & 0 & $(P>0.05)^{\mathrm{b}}$ \\
\hline
\end{tabular}

STI, sexually transmitted infection; HPV, human papillomavirus; CT, Chlamydia trachomatis; MG, Mycoplasma genitalium. ${ }^{\mathrm{a}} \mathrm{CT}$ positive cases includes: $\mathrm{CIN}=1, \mathrm{CIN}$ II $=1$ and $\operatorname{CIN} 3=5$. ${ }^{\mathrm{b}}$ There was no significant difference.

Table 2. Frequency of JAK2 polymorphism in participants

\begin{tabular}{cccc}
\hline \multicolumn{1}{c}{ Clinical subject } & & JAK2 V617F (1849 G $>$ T) & Homozygous \\
\cline { 2 - 4 } & Wild type & Heterozygous & \\
\hline Women without cervical cancer (145 cases) & & & 0 \\
HPV positive (98 cases) & $72(73.5)$ & $14(26.5)$ & 0 \\
HPV negative (47 cases) & $33(70.2)$ & $28(26.5)$ & $0(0)$ \\
Women with cervical cancer (50 cases) & $22(44.0)$ & 5 & 0 \\
CIN I (9 cases) & 4 & 4 & 0 \\
CIN II (6 cases) & 2 & 19 & 0 \\
CIN III (35 cases) & 16 & 68 (34.9) & $0(0)$ \\
Total & $127(65.1)$ & & 0 \\
\hline
\end{tabular}

Values are presented as number (\%) or number only. HPV, human papillomavirus. 
acquired mutation JAK2 V617F has been described in the majority of patients with hematologic disorders. This mutation is characterized by a $\mathrm{G}$ to $\mathrm{T}$ transverse at nucleotide 1849 in exon 12 of the JAK2 gene, located on the chromosome 9p, leading to substitution of valine to phenylalanine at amino acid position 617 in JAK2 protein. The JAK-STAT pathway is a critical tyrosine kinase modulating immune responses. However, the implication of JAK2 mutation in infectious disorders remains undetermined. Although in clinical therapeutic exposure, there is the potential of infectious complications in JAK inhibitors as JAK2 V617F kinase. The activating mutation V617F of JAK2 has been identified as one of the hallmarks in the pathogenesis of malignancies. ${ }^{15-17,20,26}$ We have not found any evidence for existence of HSV-2 and $M$. genitalium in cancer patients. It appears that $C$. trachomatis is more prevalent than HSV-2 and $M$. Genitaliumin Iran. As a result, all of cancer patients infected with HPV genotypes may be affected on these STIs. This concern should be evaluated in further studies.

The controversies in population surveys may be due to differences in the sample size, specimen's management, the genetic patterns and the assay. The results from of single nucleotide polymorphisms may be influenced by unapproved molecular assays, primer combinations, home-brew methods and multiple infections which have been effective on sensitivity and specificity of diagnostic expousers. ${ }^{27-30}$

In further epidemiological studies, whole genome and targeted sequencing of the JAK2 mutation exon 12 would be the best way for finding any correlation between the SNPs and other environmental and infection factors. Recent molecular efforts led to increased use of genetic biomarkers in diagnostic laboratories and in the surveillance system of cancers. These can be helpful for selecting the appropriate prevention strategies especially in early stage of neoplasia.

\section{ACKNOWLEDGMENTS}

We are grateful and indebted to reference health laboratory staffs and Dr. Akram Sadat Tabatabaei-Panah (assistant professor of Biology Department, East Tehran Branch, Islamic Azad University, Tehran, Iran).

\section{CONFLICTS OF INTEREST}

No potential conflicts of interest were disclosed.

\section{REFERENCES}

1. Husain RS, Ramakrishnan V. Global variation of human papillomavirus genotypes and selected genes involved in cervical malignancies. Ann Glob Health 2015;81:675-83.

2. Mboumba-Bouassa RS, Prazuck T, Lethu T, Meye JF, Bélec L. Cervical cancer in sub-Saharan Africa: an emerging and preventable disease associated with oncogenic human papillomavirus. Med Sante Trop 2017;27:16-22.

3. Shi N, Lu Q, Zhang J, Li L, Zhang J, Zhang F, et al. Analysis of risk factors for persistent infection of asymptomatic women with high-risk human papilloma virus. Hum Vaccin Immunother 2017;13:1-7.

4. Mittal S, Basu P, Muwonge R, Banerjee D, Ghosh I, Sengupta MM, et al. Risk of high-grade precancerous lesions and invasive cancers in high-risk HPV-positive women with normal cervix or CIN 1 at baseline-A population-based cohort study. Int J Cancer 2017;140:1850-9.

5. Shayanfar N, Hosseini N, Panahi M, Azadmanesh K, Mohammadpour M, Kadivar M, et al. Detection of mucosal type human papillomavirus in cutaneous squamous cell carcinoma in Iran. Pathol Res Pract 2013;209:90-4.

6. Shayanfar N, Babaheidarian P, Rahmani H, Azadmanesh K, Sohrabi A, Mohammadpour M, et al. Epidermodysplasia verruciformis associated with plasmablastic lymphoma and hepatitis B virus infection. Acta Dermatovenerol Croat 2012;20:267-71.

7. Sohrabi A, Mirab-Samiee S, Modarresi MH, Izadimood N, Azadmanesh K, Rahnamaye-Farzami M. Development of in-house multiplex real time PCR for human papillomavirus genotyping in Iranian women with cervical cancer and cervical intraepithelial neoplasia. Asian Pac J Cancer Prev 2014;15:6257-61.

8. Yasa C, Takmaz O, Dural O, Akhan SE. The value of tumor markers in endometrial carcinoma: review of literature. J Cancer Ther 2013:4:966-70.

9. Sohrabi A, Mirab-Samiee S, Rahnamaye-Farzami M, Rafizadeh M, Akhavan S, Hashemi-Bahremani M, et al. C13orf18 and C1orf166 (MULAN) DNA genes methylation are not associated with cervical cancer and precancerous lesions of human papillomavirus genotypes in Iranian women. Asian Pac J Cancer Prev 2014;15: 6745-8.

10. Zhang W, Wang L, Xin Z. Combination of serum CA19-9 and CA125 levels and contrast-enhanced ultrasound parametric data facilitates to differentiate ovarian serous carcinoma from ovarian malignant epithelial cancer. Medicine (Baltimore) 2018;97:e0358.

11. Hwang SJ, Shroyer KR. Biomarkers of cervical dysplasia and carcinoma. J Oncol 2012;2012:507286.

12. Sohrabi A, Hajia M, Jamali F, Kharazi F. Is incidence of multiple HPV genotypes rising in genital infections? J Infect Public Health 2017;10:730-3.

13. Sohrabi A, Farzami MR, Samiee MH, Modarresi MH. An overview on papillomaviruses as the main cause of cervical cancer. Iran J Obstet Gynecol Infertil 2015;18:14-25.

14. Barra F, Lorusso D, Leone Roberti Maggiore U, Ditto A, Bogani G, Raspagliesi F, et al. Investigational drugs for the treatment of cervical cancer. Expert Opin Investig Drugs 2017;26:389-402.

15. Vaclavicek A, Bermejo JL, Schmutzler RK, Sutter C, Wappenschmidt B, Meindl A, et al. Polymorphisms in the Janus kinase 2 (JAK)/signal transducer and activator of transcription 
(STAT) genes: putative association of the STAT gene region with familial breast cancer. Endocr Relat Cancer 2007; 14:267-77.

16. Zhao D, Pan C, Sun J, Gilbert C, Drews-Elger K, Azzam DJ, et al. VEGF drives cancer-initiating stem cells through VEGFR-2/Stat3 signaling to up regulate Myc and Sox2. Oncogene 2015;34: 3107-19.

17. Zhang RR, Wang H, Hui N, Zhang P. Enhanced antitumor effect of axitinib synergistic interaction with AG490 via VEGFR2/JAK2/ STAT3 signaling mediated epithelial-mesenchymal transition in cervical cancer in vitro. Asian Biomed 2013;7:39-49.

18. Zhang Z, Fye S, Borecki IB, Rader JS. Polymorphisms in immune mediators associate with risk of cervical cancer. Gynecol Oncol 2014;135:69-73.

19. Moharreri M, Sohrabi A. Herpes simplex virus 2, Mycoplasma genitalium and Chlamydia trachomatis: are they related to HPV genotypes associated with cervical cancer and genital infections? 2018 [In Press].

20. Mervat MMA, Ola AH, Mervat SHA. Detection of JAK2 mutation in cases of myelodysplastic syndrome. Zagazig Univ Med J 2015;21:79-86.

21. Dijkstra MG, Snijders PJ, Arbyn M, Rijkaart DC, Berkhof J, Meijer CJ. Cervical cancer screening: on the way to a shift from cytology to full molecular screening. Ann Oncol 2014:25:927-35.

22. Nunobiki O, Ueda M, Toji E, Yamamoto M, Akashi K, Sato N, et al. Genetic polymorphism of cancer susceptibility genes and HPV infection in cervical carcinogenesis. Patholog Res Int 2011; 2011:364069.

23. Suh DH, Kim M, Kim K, Kim HJ, Lee KH, Kim JW. Major clinical research advances in gynecologic cancer in 2016: 10-year special edition. J Gynecol Oncol 2017;28:e45.

24. Bruni L, Barrionuevo-Rosas L, Albero G, Serrano B, Mena M, Gómez D, et al. ICO Information Centre on HPV and Cancer (HPV Information Centre). www.hpvcentre.net. Accessed December, 2016.

25. Botezatu A, Socolov D, Iancu IV, Huica I, Plesa A, Ungureanu C, et al. Methylenetetrahydrofolate reductase (MTHFR) polymorphisms and promoter methylation in cervical oncogenic lesions and cancer. J Cell Mol Med 2013;17:543-49.

26. Peña G, Cai B, Deitch EA, Ulloa L. JAK2 inhibition prevents innate immune responses and rescues animals from sepsis. J Mol Med (Berl) 2010;88:851-9.

27. Sohrabi A, Rahnamaye-Farzami M, Mirab-Samiee S, Mahdavi S, Babaei M. Comparison of in-house multiplex real time PCR, Diagcor GenoFlow HPV array test and INNO-LiPA HPV genotyping extra assays with LCD- array kit for human papillomavirus genotyping in cervical liquid based cytology specimens and genital lesions in Tehran, Iran. Clin Lab 2016;62:615-9.

28. Arbyn M, Ronco G, Cuzick J, Wentzensen N, Castle PE. How to evaluate emerging technologies in cervical cancer screening? Int J Cancer 2009;125:2489-96.

29. Sohrabi A, Hajia M. Cervical cancer and genital infections: assessment of performance and validation in human papillomavirus genotyping assays in Iran, its neighbouring countries and Persian gulf area. Iran J Pathol 2017;12:35-44.

30. Hajia M, Sohrabi A. Possible synergistic interactions among multiple HPV genotypes in women suffering from genital neoplasia. Asian Pac J Cancer Prev 2018;19:785-9. 